\title{
Equivalent and Alternative Forms for BF Gravity with Immirzi Parameter ${ }^{\star}$
}

\author{
Merced MONTESINOS and Mercedes VELÁZQUEZ \\ Departamento de Física, Cinvestav, Instituto Politécnico Nacional 2508, \\ San Pedro Zacatenco, 07360, Gustavo A. Madero, Ciudad de México, México \\ E-mail: merced@fis.cinvestav.mx,mquesada@fis.cinvestav.mx \\ URL: http://www.fis.cinvestav.mx/ merced/
}

Received August 31, 2011, in final form November 07, 2011; Published online November 11, 2011 http://dx.doi.org/10.3842/SIGMA.2011.103

\begin{abstract}
A detailed analysis of the BF formulation for general relativity given by Capovilla, Montesinos, Prieto, and Rojas is performed. The action principle of this formulation is written in an equivalent form by doing a transformation of the fields of which the action depends functionally on. The transformed action principle involves two BF terms and the two Lorentz invariants that appear in the original action principle generically. As an application of this formalism, the action principle used by Engle, Pereira, and Rovelli in their spin foam model for gravity is recovered and the coupling of the cosmological constant in such a formulation is obtained.
\end{abstract}

Key words: BF theory; BF gravity; Immirzi parameter; Holst action

2010 Mathematics Subject Classification: 83C05; 83C45

\section{Introduction}

One of the main challenges nowadays is to establish links between loop quantum gravity (LQG) [1] and spin foam models [2], which are the main approaches to the nonperturbative and background-independent quantizations of general relativity. Whether or not the two quantization schemes yield two different quantum theories is still an open problem (see [3]). The search for the links between these approaches lies mostly in the quantum realm, but there are still some aspects of this correspondence that are unclear classically. It is possible to say that the connection between the two frameworks at the classical level is the relationship between the Holst's action and the BF formulations for general relativity because LQG is based at the classical level on Holst's action but spin foam models for gravity are related to constrained BF theories. This is the issue studied in this paper.

General relativity expressed as a constrained BF theory was given by Plebański many years ago [4]. The basic idea behind the Plebański formulation is that the fundamental variables for describing the gravitational field (general relativity) are neither a metric (as it is in the Einstein-Hilbert action) nor a tetrad together with a Lorentz connection (as it is in the Palatini action), but rather two-form fields, a connection one-form, and some Lagrange multipliers. The geometry of spacetime is built up from these fundamental blocks. In order to bring tetrads into the formalism, the two-forms are eliminated by solving an equation among them, which implies that the two-forms can be expressed in terms of tetrad fields, and by inserting back this expression for the two-forms into the Plebański action, it becomes the self-dual action for general relativity $[5,6]$.

\footnotetext{
*This paper is a contribution to the Special Issue "Loop Quantum Gravity and Cosmology". The full collection is available at http://www.emis.de/journals/SIGMA/LQGC.html
} 
This view point has been adopted in the construction of other action principles, which also express general relativity as a constrained BF theory [7, 8, 9, 10, 11, 12]. The link with tetrad gravity is again made by solving the constraints for the two-form fields which amounts to express them in terms of tetrad fields. In particular, a formulation for real general relativity expressed as a constrained BF theory that involves the Immirzi parameter $[13,14,15]$ was given in [12] by Capovilla, Montesinos, Prieto, and Rojas (hereafter CMPR formulation). It is well-known that the Immirzi parameter in such a formulation appears naturally when the two Lorentz invariants $\eta_{I K} \eta_{J L}-\eta_{I L} \eta_{J K}$ and $\varepsilon_{I J K L}$ are introduced in the constraint on the Lagrange multipliers $\phi_{I J K L}$. This action principle involves just one BF term. In this work it is shown that by performing a suitable transformation on the fields involved in the theory, the original action principle can be written in a form that involves two BF terms, one of them containing a parameter that will be identified a posteriori with the Immirzi parameter (see also [16]). This allows us to relate the CMPR formulation with different real BF formulations of gravity currently employed in the literature (see e.g. [17]). Furthermore, the same transformation is applied to the action principle studied in [18], which includes the cosmological constant, and we obtain the coupling of the cosmological constant in the framework of [17]. The material reported in this paper is part of the work presented in [19].

\section{CMPR action for gravity}

The action principle for pure gravity introduced by Capovilla, Montesinos, Prieto, and Rojas in [12] is given by

$$
\begin{aligned}
S[Q, A, \psi, \mu]=\int_{\mathcal{M}^{4}}[ & Q^{I J} \wedge F_{I J}[A]-\frac{1}{2} \psi_{I J K L} Q^{I J} \wedge Q^{K L} \\
& \left.-\mu\left(a_{1} \psi_{I J}^{I J}+a_{2} \psi_{I J K L} \varepsilon^{I J K L}\right)\right],
\end{aligned}
$$

where $A^{I}{ }_{J}$ is an Euclidean or Lorentz connection one-form, depending on whether $S O(4)$ or $S O(3,1)$ is taken as the internal gauge group, and $F^{I}{ }_{J}[A]=d A^{I}{ }_{J}+A^{I}{ }_{K} \wedge A^{K}{ }_{J}$ is its curvature; the $Q$ 's are a set of six two-forms on account of their antisymmetry $Q^{I J}=-Q^{J I}$; the Lagrange multiplier $\psi_{I J K L}$ has 21 independent components due to the properties $\psi_{I J K L}=\psi_{K L I J}$, $\psi_{I J K L}=-\psi_{J I K L}$, and $\psi_{I J K L}=-\psi_{I J L K}$; the Lagrange multiplier $\mu$ implies the additional restriction $a_{1} \psi_{I J}^{I J}+a_{2} \psi_{I J K L} \varepsilon^{I J K L}=0$ on the Lagrange multiplier $\psi_{I J K L}$. The Lorentz (Euclidean) indices $I, J, K, \ldots=0,1,2,3$ are raised and lowered with the Minkowski (Euclidean) metric $\left(\eta_{I J}\right)=\operatorname{diag}(\sigma,+1,+1,+1)$ where $\sigma=+1$ for Euclidean and $\sigma=-1$ for Lorentzian signatures, respectively.

The variation of the action (1) with respect to the independent fields gives the equations of motion

$$
\begin{aligned}
& \delta Q: F_{I J}[A]-\psi_{I J K L} Q^{K L}=0, \\
& \delta A: D Q^{I J}=0, \\
& \delta \psi: Q^{I J} \wedge Q^{K L}+2 a_{1} \mu \eta^{[I|K|} \eta^{J] L}+2 a_{2} \mu \varepsilon^{I J K L}=0, \\
& \delta \mu: a_{1} \psi_{I J}^{I J}+a_{2} \psi_{I J K L} \varepsilon^{I J K L}=0 .
\end{aligned}
$$

By contracting equation (2) with the Killing-Cartan metric $\eta_{[I|K|} \eta_{J] L}=\frac{1}{2}\left(\eta_{I K} \eta_{J L}-\eta_{I L} \eta_{J K}\right)$ and $\varepsilon_{I J K L}$, one gets $a_{1} \mu=-\frac{1}{12} Q^{I J} \wedge Q_{I J}$ and $a_{2} \mu=-\frac{\sigma}{4 !} Q^{I J} \wedge{ }^{*} Q_{I J}$ respectively, where ${ }^{*} Q^{I J}:=\frac{1}{2} \varepsilon^{I J}{ }_{K L} Q^{K L}$. The non-degenerate case corresponds to $\mu \neq 0$ whereas the degenerate case corresponds to $\mu=0$. Let us restrict the analysis to the non-degenerate case. Inserting back $a_{1} \mu$ and $a_{2} \mu$ into (2), it is obtained

$$
Q^{I J} \wedge Q^{K L}-\frac{1}{6}\left(Q^{M N} \wedge Q_{M N}\right) \eta^{[I|K|} \eta^{J] L}-\frac{2 \sigma}{4 !}\left(Q^{M N} \wedge{ }^{*} Q_{M N}\right) \varepsilon^{I J K L}=0,
$$


together with

$$
2 a_{2} Q^{I J} \wedge Q_{I J}-\sigma a_{1} Q^{I J} \wedge^{*} Q_{I J}=0
$$

that follows from the equality of the two expressions for $\mu$ and the fact that $a_{1} \neq 0$ and $a_{2} \neq 0$. It is shown in [12] that

$$
Q^{I J}=\alpha^{*}\left(e^{I} \wedge e^{J}\right)+\beta e^{I} \wedge e^{J},
$$

is the general solution for the $Q$ 's provided that the constants $\alpha$ and $\beta$ satisfy

$$
\frac{a_{2}}{a_{1}}=\frac{\alpha^{2}+\sigma \beta^{2}}{4 \alpha \beta} .
$$

By inserting the solution (3) into the action principle (1), we get

$$
S[e, A]=\int_{\mathcal{M}^{4}}\left[*\left(e^{I} \wedge e^{J}\right)+\frac{\beta}{\alpha} e^{I} \wedge e^{J}\right] \wedge F_{I J}[A] .
$$

Notice that, as remarked in [12], the Immirzi parameter appears naturally in equation (3) because the two invariants $\psi_{I J}^{I J}$ and $\psi_{I J K L} \varepsilon^{I J K L}$ are present in the action.

\subsection{CMPR formulation with $a_{1}=0$ or $a_{2}=0$}

The cases when $a_{1}$ or $a_{2}$ are equal to zero have been analyzed separately [8,10,11]. In particular, if $a_{1}=0$ and $a_{2} \neq 0$ the Lorentz invariant $\psi_{I J}{ }^{I J}$ is not present in the action, which reduces to

$$
S[Q, A, \psi, \mu]=\int_{\mathcal{M}^{4}}\left[Q^{I J} \wedge F_{I J}[A]-\frac{1}{2} \psi_{I J K L} Q^{I J} \wedge Q^{K L}-\mu \psi_{I J K L} \varepsilon^{I J K L}\right] .
$$

After solving the constraint on the $Q$ 's, they can be written in terms of the tetrad $e^{I}$ as

$$
\text { (i) } \quad Q^{I J}=\kappa_{1}^{*}\left(e^{I} \wedge e^{J}\right), \quad \text { (ii) } \quad Q^{I J}=\kappa_{2} e^{I} \wedge e^{J}
$$

where $\kappa_{1}, \kappa_{2}$ are constants. By inserting these expressions for the $Q$ 's into (5), we get action principles for two different theories, one of which is general relativity $[10,11]$

$$
\begin{aligned}
& \text { (i) } \quad S_{1}[e, A]=\kappa_{1} \int_{\mathcal{M}^{4}} *\left(e^{I} \wedge e^{J}\right) \wedge F_{I J}[A], \\
& \text { (ii) } \quad S_{2}[e, A]=\kappa_{2} \int_{\mathcal{M}^{4}}\left(e^{I} \wedge e^{J}\right) \wedge F_{I J}[A] .
\end{aligned}
$$

In [20] was proposed to consider the action $S_{2}[e, A]$ as a genuine field theory in its own right because it is background-independent and diffeomorphism-invariant. Recently, it has been shown that $S_{2}[e, A]$ is indeed topological if the spacetime $\mathcal{M}^{4}$ has no boundary. The proof is given by performing the covariant canonical analysis to this action as well as by doing the Dirac's canonical analysis with and without breaking local Lorentz invariance [21, 22, 23]. The relevance of the action $S_{2}[e, A]$ is not academic, this field theory is a topological limit of general relativity obtained by taking the Newton constant $G \rightarrow \infty$ and the Immirzi parameter $\gamma \rightarrow 0$ while keeping the product $G \gamma$ constant [21].

On the other hand, if $a_{1} \neq 0$ and $a_{2}=0$, the action takes the form [8]

$$
S[Q, A, \psi, \mu]=\int_{\mathcal{M}^{4}}\left[Q^{I J} \wedge F_{I J}[A]-\frac{1}{2} \psi_{I J K L} Q^{I J} \wedge Q^{K L}-\mu \psi_{I J} I J\right] .
$$


The solutions for the two-forms in terms of the tetrad $e^{I}$ are given by

$$
Q^{I J}=\kappa\left[{ }^{*}\left(e^{I} \wedge e^{J}\right) \pm \sqrt{-\sigma} e^{I} \wedge e^{J}\right] .
$$

Therefore, the action principle in terms of the tetrad takes the form

$$
S_{E}[e, A]=\kappa \int_{\mathcal{M}^{4}}\left[*\left(e^{I} \wedge e^{J}\right) \pm i e^{I} \wedge e^{J}\right] \wedge F_{I J}[A]
$$

in the Euclidean case whereas in the Lorentzian case it becomes

$$
S_{L}[e, A]=\kappa \int_{\mathcal{M}^{4}}\left[*\left(e^{I} \wedge e^{J}\right) \pm e^{I} \wedge e^{J}\right] \wedge F_{I J}[A] .
$$

Notice that last form of the action includes the coupling of the term $e^{I} \wedge e^{J} \wedge F_{I J}[A]$ added by Holst to the Palatini action with (what it is called now) Immirzi parameter equal to \pm 1 , but this form of the action was reported in [8] several years before Holst, Immirzi, and Barbero's papers.

\section{Transformation of the CMPR action for gravity}

The goal of this section is to study the CMPR formulation for general relativity by performing a linear transformation from the original variables the action principle depends functionally on to a new set of two-forms and Lagrange multipliers. It will be shown that the resulting action principle involves the two possible BF terms, $B^{I J} \wedge F_{I J}[A]$ and ${ }^{*} B^{I J} \wedge F_{I J}[A]$ that can be built when the internal gauge group is $S O(3,1)$ or $S O(4)$, with the corresponding change in the symplectic structure as it was pointed out in [24] and [25].

In order to do what we have explained, i.e., the alternative writing of the CMPR action, we define

$$
Q^{I J}:=b_{1} B^{I J}+b_{2}{ }^{*} B^{I J},
$$

with $b_{1}$ and $b_{2}$ constants, from which it follows the inverse transformation

$$
B^{I J}=\frac{1}{b_{1}^{2}-\sigma b_{2}^{2}}\left(b_{1} Q^{I J}-b_{2}{ }^{*} Q^{I J}\right),
$$

provided that

$$
b_{1}^{2}-\sigma b_{2}^{2} \neq 0,
$$

holds.

Using (9), the Lagrangian of action principle (1) acquires the form

$$
\begin{aligned}
& \left(b_{1} B^{I J}+b_{2}{ }^{*} B^{I J}\right) \wedge F_{I J}[A]-\frac{1}{2} \psi_{I J K L}\left(b_{1} B^{I J}+b_{2}{ }^{*} B^{I J}\right) \wedge\left(b_{1} B^{K L}-b_{2}{ }^{*} B^{K L}\right) \\
& -\mu\left(a_{1} \psi_{I J}{ }^{I J}+a_{2} \psi_{I J K L} \varepsilon^{I J K L}\right),
\end{aligned}
$$

that can further be rewritten by defining

$$
\phi_{I J K L}:=b_{1}^{2} \psi_{I J K L}+b_{1} b_{2}{ }^{*} \psi_{I J K L}+b_{1} b_{2} \psi_{I J K L}^{*}+b_{2}^{2 *} \psi_{I J K L}^{*},
$$

where ${ }^{*} \psi_{I J K L}:=\frac{1}{2} \varepsilon^{M N}{ }_{I J} \psi_{M N K L}$ and $\psi_{I J K L}^{*}:=\frac{1}{2} \varepsilon^{M N}{ }_{K L} \psi_{I J M N}$ are the dual on the first and on the second pair of Lorentz indices, respectively. From (13) it follows that

$$
\psi_{I J K L}=\frac{1}{\left(b_{1}^{2}-\sigma b_{2}^{2}\right)^{2}}\left(b_{1}^{2} \phi_{I J K L}-b_{1} b_{2}{ }^{*} \phi_{I J K L}-b_{1} b_{2} \phi^{*}{ }_{I J K L}+b_{2}^{2 *} \phi_{I J K L}^{*}\right) .
$$


Therefore, the second term of (12) takes the form

$$
\psi_{I J K L}\left(b_{1} B^{I J}+b_{2}{ }^{*} B^{I J}\right) \wedge\left(b_{1} B^{K L}-b_{2}{ }^{*} B^{K L}\right)=\phi_{I J K L} B^{I J} \wedge B^{K L} .
$$

Furthermore, using (14), the two invariants $\psi_{I J}^{I J}$ and $\psi_{I J K L} \varepsilon^{I J K L}$ can be written in terms of the two invariants $\phi_{I J}^{I J}$ and $\varepsilon_{I J K L} \phi^{I J K L}$ as

$$
\psi_{I J}^{I J}=\frac{1}{\left(b_{1}^{2}-\sigma b_{2}^{2}\right)^{2}}\left[\left(b_{1}^{2}+\sigma b_{2}^{2}\right) \phi_{I J}^{I J}-b_{1} b_{2} \phi_{I J K L} \varepsilon^{I J K L}\right],
$$

and

$$
\psi_{I J K L} \varepsilon^{I J K L}=\frac{1}{\left(b_{1}^{2}-\sigma b_{2}^{2}\right)^{2}}\left[\left(b_{1}^{2}+\sigma b_{2}^{2}\right) \phi_{I J K L} \varepsilon^{I J K L}-4 \sigma b_{1} b_{2} \phi_{I J}^{I J}\right] .
$$

Thus, using (15) and (16), the last term of (12) acquires the form

$$
a_{1} \psi_{I J}^{I J}+a_{2} \psi_{I J K L} \varepsilon^{I J K L}=A_{1} \phi_{I J}^{I J}+A_{2} \phi_{I J K L} \varepsilon^{I J K L},
$$

with

$$
\begin{aligned}
& A_{1}=\frac{1}{\left(b_{1}^{2}-\sigma b_{2}^{2}\right)^{2}}\left[a_{1}\left(b_{1}^{2}+\sigma b_{2}^{2}\right)-4 \sigma a_{2} b_{1} b_{2}\right], \\
& A_{2}=\frac{1}{\left(b_{1}^{2}-\sigma b_{2}^{2}\right)^{2}}\left[a_{2}\left(b_{1}^{2}+\sigma b_{2}^{2}\right)-a_{1} b_{1} b_{2}\right] .
\end{aligned}
$$

By using the previous steps, the form that the CMPR action (1) acquires once the transformation defined in equations (9), (10) and in equations (13), (14) has been done is

$$
\begin{aligned}
S[B, A, \phi, \mu]=\int_{\mathcal{M}^{4}}[ & \left(b_{1} B^{I J}+b_{2}{ }^{*} B^{I J}\right) \wedge F_{I J}[A]-\frac{1}{2} \phi_{I J K L} B^{I J} \wedge B^{K L} \\
& \left.-\mu\left(A_{1} \phi_{I J}{ }^{I J}+A_{2} \phi_{I J K L} \varepsilon^{I J K L}\right)\right] .
\end{aligned}
$$

Due to the fact that the transformation is invertible, both actions (1) and (19) are equivalent.

The action principle (19) can still be written in terms of tetrads and a Lorentz connection by solving the constraint on the $B$ 's coming from it. Alternatively, the expression for the twoforms $B^{I J}$ in terms of the tetrad field can be obtained from the expression for the $Q$ 's given in (3) and from the use of the equation (10).

The relationship between the action principle (1) and (19) will be analyzed in detail in an example given in Section 3.1. Some remarks follow:

(a) It can be observed from equations (15), (16), (17), and (18) that even though we had started from action (1) with either $a_{1}=0$ or $a_{2}=0$, it might be possible to obtain generically the two Lorentz invariants in the transformed action (19).

(b) It is possible to get just one of the invariants in the transformed action (19) by imposing either $A_{1}=0$ or $A_{2}=0$. For instance, the case $A_{1}=0$ can be achieved by solving for the ratio $b_{2} / b_{1}$ in terms of the ratio $a_{2} / a_{1}$, i.e. by choosing a particular transformation (encoded in $b_{1}$ and $b_{2}$ ) and leaving $a_{1}$ and $a_{2}$ arbitrary. Alternatively, $A_{1}=0$ can be achieved by solving for the ratio $a_{2} / a_{1}$ in terms of the ratio $b_{2} / b_{1}$, i.e. by choosing a particular form for the ratio $a_{2} / a_{1}$ and leaving the transformation arbitrary. Similarly, the case $A_{2}=0$ can also be handled in two analogous ways. 
The previous analysis points out that is not correct to refer to the term ${ }^{*} B^{I J} \wedge F_{I J}$ as "Holst's term" simply because the term added by Holst to the Palatini action and given by $e^{I} \wedge e^{J} \wedge F_{I J}$ [15] (see also [8]) is at the level of tetrads $e^{I}$ and not at the level of BF theories. Even though they might be related, they are not exactly the same thing. In particular, ${ }^{*} B^{I J} \wedge F_{I J}$ could be proportional to ${ }^{*}\left(e^{I} \wedge e^{J}\right) \wedge F_{I J}$, or to $e^{I} \wedge e^{J} \wedge F_{I J}$, or to something else depending on the expression for the $B$ 's that solves the constraint among them, i.e., a priori there is not guarantee that ${ }^{*} B^{I J} \wedge F_{I J}$ would lead to the term added by Holst, because this will ultimately depend on the expression for the $B$ 's in terms of the tetrads.

\subsection{A particular transformation}

Let us now study a particular case of the transformation (9). Taking $b_{1}=1$ and $b_{2}=\frac{1}{\gamma}$ the transformation is invertible for $\gamma^{2} \neq \sigma$. In this case, the action (1) takes the form

$$
\begin{aligned}
S[B, A, \phi, \mu]=\int_{\mathcal{M}^{4}} & {\left[\left(B^{I J}+\frac{1}{\gamma}{ }^{*} B^{I J}\right) \wedge F_{I J}[A]-\frac{1}{2} \phi_{I J K L} B^{I J} \wedge B^{K L}\right.} \\
& \left.-\mu\left(A_{1} \phi_{I J}^{I J}+A_{2} \phi_{I J K L} \varepsilon^{I J K L}\right)\right]
\end{aligned}
$$

where now

$$
A_{1}=\frac{\gamma^{2}}{\left(\gamma^{2}-\sigma\right)^{2}}\left[a_{1}\left(\gamma^{2}+\sigma\right)-4 \sigma a_{2} \gamma\right], \quad A_{2}=\frac{\gamma^{2}}{\left(\gamma^{2}-\sigma\right)^{2}}\left[a_{2}\left(\gamma^{2}+\sigma\right)-a_{1} \gamma\right] .
$$

It is important to notice that, at this stage, the action principle (20) is completely equivalent to action (1) because the coefficients of the transformation satisfy the condition (11).

As pointed out in the previous remark (b), it is possible to obtain only one of the invariants in the action (20) by imposing, additionally, either $A_{1}=0$ or $A_{2}=0$.

\subsubsection{Case $A_{1}=0$ : action with the invariant $\phi_{I J K L} \varepsilon^{I J K L}$ only}

In order to eliminate the term with $\phi_{I J}{ }^{I J}$ in the action (20), $A_{1}$ must vanish; this is only possible if $a_{2}$ and $a_{1}$ satisfy the condition

$$
\frac{a_{2}}{a_{1}}=\frac{\gamma^{2}+\sigma}{4 \gamma \sigma}
$$

Using (22), the CMPR action principle (1) takes the form

$$
\begin{aligned}
S[Q, A, \psi, \mu]=\int_{\mathcal{M}^{4}} & {\left[Q^{I J} \wedge F_{I J}[A]-\frac{1}{2} \psi_{I J K L} Q^{I J} \wedge Q^{K L}\right.} \\
& \left.-\mu a_{1}\left(\psi_{I J}^{I J}+\frac{\gamma^{2}+\sigma}{4 \gamma \sigma} \psi_{I J K L} \varepsilon^{I J K L}\right)\right],
\end{aligned}
$$

whereas the transformed action principle (20) becomes

$$
\begin{aligned}
S[B, A, \phi, \mu]=\int_{\mathcal{M}^{4}} & {\left[\left(B^{I J}+\frac{1}{\gamma}{ }^{*} B^{I J}\right) \wedge F_{I J}[A]-\frac{1}{2} \phi_{I J K L} B^{I J} \wedge B^{K L}\right.} \\
& \left.-\mu \frac{a_{1} \sigma \gamma}{4} \phi_{I J K L} \varepsilon^{I J K L}\right] .
\end{aligned}
$$

This is the form of the action principle used in [17] (see also [16]). 


\section{Remarks:}

(c) In the Lorentzian case $\sigma=-1$, the particular values $\gamma= \pm 1$ imply $\gamma^{2}+\sigma=0$ and thus the Lorentz invariant $\phi_{I J K L} \varepsilon^{I J K L}$ is not present in the action (23), which then reduces to the form given in (8). This means that the action (24) can be written as the action studied in [8] by taking $\gamma= \pm 1$ in the Lorentzian case. For any other arbitrary real value of the Immirzi parameter the two invariants $\psi_{I J}^{I J}$ and $\psi_{I J K L} \varepsilon^{I J K L}$ are present in the action (23) [12].

(d) In the Euclidean case, $\sigma=1$, for real values of $\gamma$ it follows that $\gamma^{2}+\sigma \neq 0$ and therefore the two Lorentz invariants are always present in the action (23). This means that the two invariants $\psi_{I J}^{I J}$ and $\psi_{I J K L} \varepsilon^{I J K L}$ must be involved in order to include arbitrary real values of $\gamma$, as it was recognized in [12]. Nevertheless, it is important to notice that $\gamma^{2}+\sigma$ can vanish if complex values of $\gamma$ are allowed, $\gamma= \pm i$. For these values the invariant $\psi_{I J K L} \varepsilon^{I J K L}$ is missing in the action (23), which becomes also the one given in (8).

Continuing with the analysis, the expression for the $B$ 's can be directly obtained from action principle (24). However, it can be alternatively obtained from the $Q$ 's given in (3) and (4) (and supplemented with (22)) and from the use of the inverse transformation (10) with $b_{1}=1$ and $b_{2}=\frac{1}{\gamma}$. We are going to follow this last approach. Therefore, from the equality of equations (4) and (22) it follows that in order for the $Q$ 's in (3) to be solutions for the action principle (23), $\alpha / \beta$ must satisfy the quadratic equation

$$
\left(\frac{\alpha}{\beta}\right)^{2}-\left(\frac{1}{\gamma}+\sigma \gamma\right) \frac{\alpha}{\beta}+\sigma=0
$$

whose solutions are

$$
\text { (i) } \quad \alpha / \beta=\sigma \gamma, \quad \text { and } \quad(\text { ii }) \quad \alpha / \beta=\frac{1}{\gamma} .
$$

The first root was explicitly mentioned in [12], but the second one was not recognized there as a possibility to include the Immirzi parameter.

Inserting the two roots given in (25) into (3), we get the corresponding expression for the $Q$ 's

$$
\text { (i) } \quad Q^{I J}=\alpha\left[*\left(e^{I} \wedge e^{J}\right)+\frac{\sigma}{\gamma} e^{I} \wedge e^{J}\right], \quad \text { (ii) } \quad Q^{I J}=\alpha\left[*\left(e^{I} \wedge e^{J}\right)+\gamma e^{I} \wedge e^{J}\right],
$$

and by plugging them into $(10)$ with the restrictions $b_{1}=1$ and $b_{2}=\frac{1}{\gamma}$ we get the corresponding expressions for the $B$ 's

$$
\text { (i) } \quad B^{I J}=\alpha^{*}\left(e^{I} \wedge e^{J}\right), \quad(i i) \quad B^{I J}=\alpha \gamma e^{I} \wedge e^{J},
$$

which are precisely the ones given in (6). Furthermore, by comparing (6) and (27), we conclude that $\kappa_{1}=\alpha$ for $(i)$ (and thus $\kappa_{1}=\sigma \gamma \beta$ ) whereas $\kappa_{2}=\alpha \gamma$ for $(i i)$ (and thus $\kappa_{2}=\beta$ ).

By plugging (27) into (24) or, equivalently, by plugging (26) into (23), we get

$$
\begin{aligned}
& \text { (i) } S_{1}[e, A]=\kappa_{1} \int_{\mathcal{M}^{4}}\left[*\left(e^{I} \wedge e^{J}\right)+\frac{\sigma}{\gamma} e^{I} \wedge e^{J}\right] \wedge F_{I J}[A], \\
& \text { (ii) } S_{2}[e, A]=\frac{\kappa_{2}}{\gamma} \int_{\mathcal{M}^{4}}\left[*\left(e^{I} \wedge e^{J}\right)+\gamma e^{I} \wedge e^{J}\right] \wedge F_{I J}[A],
\end{aligned}
$$

which is exactly the same result that we had obtained if we had directly solved the constraint on the $B$ 's that comes from (24) [17]. It is common to take $\kappa_{1}= \pm 1$ and $\kappa_{2}= \pm 1$. Nevertheless, it must be stressed that these values do not come out from the sole handling of the equations of motion. 


\subsubsection{Case $A_{2}=0$ : action with the invariant $\phi_{I J} I J$ only}

In order to obtain the action (24) from (20), a particular function for the ratio $a_{2} / a_{1}$ has been taken such that $A_{1}$ in equation (21) vanishes once the transformation is performed. So, it is natural to ask what happens if, instead of $A_{1}$, it is the coefficient $A_{2}$ which is forced to vanish in such a way that the invariant $\phi_{I J K L} \varepsilon^{I J K L}$ is not present in the action (20). From (21) this condition is equivalent to

$$
\frac{a_{1}}{a_{2}}=\frac{\gamma^{2}+\sigma}{\gamma}
$$

This means that starting from the CMPR action principle in the form

$$
\begin{aligned}
S[Q, A, \psi, \mu]=\int_{\mathcal{M}^{4}} & {\left[Q^{I J} \wedge F_{I J}[A]-\frac{1}{2} \psi_{I J K L} Q^{I J} \wedge Q^{K L}\right.} \\
& \left.-\mu a_{2}\left(\frac{\gamma^{2}+\sigma}{\gamma} \psi_{I J}^{I J}+\psi_{I J K L} \varepsilon^{I J K L}\right)\right],
\end{aligned}
$$

and using the transformation (10) with $b_{1}=1$ and $b_{2}=1 / \gamma$, this action principle acquires the form

$$
S[B, A, \phi, \mu]=\int_{\mathcal{M}^{4}}\left[\left(B^{I J}+\frac{1}{\gamma}{ }^{*} B^{I J}\right) \wedge F_{I J}[A]-\frac{1}{2} \phi_{I J K L} B^{I J} \wedge B^{K L}-\mu a_{2} \gamma \phi_{I J}^{I J}\right] .
$$

Remarks:

(e) In the case $\gamma^{2}+\sigma \neq 0$ it follows from (29) that the two Lorentz invariants are present in the action and therefore the expression for the two-forms $Q$ 's in terms of the tetrad field, and thus the form of the $B$ 's in (30), can be obtained from (3) following the same procedure carried out in the previous section. In this case the value of $a_{2} / a_{1}$ comes from (28) and the final form of the actions (29) and (30) in terms of the tetrad field and the connection are

$$
\begin{aligned}
& \text { (i) } S_{1}[e, A]=\alpha \int_{\mathcal{M}^{4}}\left[*\left(e^{I} \wedge e^{J}\right)+\sqrt{-\sigma} \frac{\gamma-\sqrt{-\sigma}}{\gamma+\sqrt{-\sigma}} e^{I} \wedge e^{J}\right] \wedge F_{I J}[A], \\
& \text { (ii) } S_{2}[e, A]=\alpha \int_{\mathcal{M}^{4}}\left[*\left(e^{I} \wedge e^{J}\right)-\sqrt{-\sigma} \frac{\gamma+\sqrt{-\sigma}}{\gamma-\sqrt{-\sigma}} e^{I} \wedge e^{J}\right] \wedge F_{I J}[A],
\end{aligned}
$$

corresponding to the two solutions for the $B$ 's.

(f) In the case $\gamma^{2}+\sigma=0$ the Lorentz invariant $\psi_{I J}{ }^{I J}$ is missing in the action (29) and it becomes the one given in (5). In that analysis the action principles (7) were obtained by plugging in (5) the solution for the $Q$ 's. In a similar way, the action principle (30) restricted to $\gamma= \pm \sqrt{-\sigma}$ can be written in terms of the tetrad field by solving for the $B$ 's and plugging the solutions into (30). The resulting action principles obtained following one or the other procedure are different by a global factor.

\section{Application of the transformation to the coupling of the cosmological constant}

The coupling of the cosmological constant to the CMPR action principle was done in [18] (see also [26]). However, taking into account the previous analysis, it is natural to wonder how the coupling looks like in the transformed CMPR action principles discussed in Section 3. In 
particular we are interested in the action principle analyzed in Section 3.1.1 and used in [17]. To do this task, the starting point is the CMPR action principle coupled with the cosmological constant given in [18]. This action principle can be transformed to one with two $\mathrm{BF}$ terms by applying the general transformation encoded in equations (9) and (14). This will give us an equivalent action principle for gravity with cosmological constant. Nevertheless, as we are interested in the coupling of the cosmological constant to gravity in an action principle of the form given in (24), i.e. with the linear combination $B^{I J}+\frac{1}{\gamma}{ }^{*} B^{I J}$ and using only the Lorentz invariant $\phi_{I J K L} \varepsilon^{I J K L}$, it is necessary to apply the particular transformation studied in Section 3 (defined by $b_{1}=1$ and $b_{2}=\frac{1}{\gamma}$ ), and to restrict the constants included in the CMPR action principle to those which lead us to (24), i.e. to impose on $a_{1}$ and $a_{2}$ the condition (22).

The action principle introduced in [18] is given by

$$
\begin{aligned}
S[Q, A, \psi, \mu]= & \int_{\mathcal{M}^{4}}\left[Q^{I J} \wedge F_{I J}[A]-\frac{1}{2} \psi_{I J K L} Q^{I J} \wedge Q^{K L}\right. \\
& \left.-\mu\left(a_{1} \psi_{I J}{ }^{I J}+a_{2} \psi_{I J K L} \varepsilon^{I J K L}-\lambda\right)+l_{1} Q_{I J} \wedge Q^{I J}+l_{2} Q_{I J} \wedge^{*} Q^{I J}\right],
\end{aligned}
$$

with $\lambda=a_{1}\left(4 ! l_{2} \sigma \frac{a_{2}}{a_{1}}+12 l_{1}+\frac{\Lambda}{\beta}\right)$. It is important to mention that this expression for $\lambda$ comes from the fact that $\Lambda$ is identified with the cosmological constant in the tetrad formalism (see [18] for the details).

By applying the transformation given in equation (9), the action principle (31) takes the form

$$
\begin{aligned}
S[B, A, \phi, \mu]= & \int_{\mathcal{M}^{4}}\left[\left(b_{1} B^{I J}+b_{2}{ }^{*} B^{I J}\right) \wedge F_{I J}[A]-\frac{1}{2} \phi_{I J K L} B^{I J} \wedge B^{K L}\right. \\
& \left.-\mu\left(A_{1} \phi_{I J}^{I J}+A_{2} \phi_{I J K L} \varepsilon^{I J K L}-\lambda\right)+K_{1} B_{I J} \wedge B^{I J}+K_{2} B_{I J} \wedge^{*} B^{I J}\right],
\end{aligned}
$$

where the constants $A_{1}$ and $A_{2}$ are given by equation (18) and

$$
K_{1}=l_{1}\left(b_{1}^{2}+\sigma b_{2}^{2}\right)+2 l_{2} b_{1} b_{2} \sigma, \quad K_{2}=l_{2}\left(b_{1}^{2}+\sigma b_{2}^{2}\right)+2 l_{1} b_{1} b_{2} .
$$

Note that the last two terms of equation (32) come from the analogous terms that appear in equation (31). These terms could still be removed by doing a redefinition of the Lagrange multiplier $\phi_{I J K L} \rightarrow \varphi_{I J K L}$ but this would imply a transformation $\psi_{I J K L} \rightarrow \varphi_{I J K L}$ different from the one given in (13). Such a transformation would be an example of how we can choose the transformation depending on the action principles we want to relate. Nevertheless, in this section we are only interested in an application of the transformation introduced in Section 3, therefore we will continue with the original transformation (13).

Notice that $\lambda$ can be written in terms of $K_{1}$ and $K_{2}$ as

$$
\lambda=12 A_{1} K_{1}+4 ! \sigma A_{2} K_{2}+\frac{\Lambda a_{1}}{\beta} .
$$

Let us now take the particular transformation $b_{1}=1$ and $b_{2}=\frac{1}{\gamma}$ used in Section 3.1.1. In this case, the action (32) takes on the form

$$
\begin{aligned}
S[B, A, \phi, \mu]= & \int_{\mathcal{M}^{4}}\left[\left(B^{I J}+\frac{1}{\gamma}{ }^{*} B^{I J}\right) \wedge F_{I J}[A]-\frac{1}{2} \phi_{I J K L} B^{I J} \wedge B^{K L}\right. \\
& \left.-\mu\left(A_{1} \phi_{I J}^{I J}+A_{2} \phi_{I J K L} \varepsilon^{I J K L}-\lambda\right)+K_{1} B_{I J} \wedge B^{I J}+K_{2} B_{I J} \wedge^{*} B^{I J}\right],
\end{aligned}
$$

where now $A_{1}$ and $A_{2}$ are given by (21), and (33) reduces to

$$
K_{1}=l_{1} \frac{\left(\gamma^{2}+\sigma\right)^{2}}{\gamma^{2}}+2 l_{2} \frac{\sigma}{\gamma}, \quad K_{2}=l_{2} \frac{\left(\gamma^{2}+\sigma\right)^{2}}{\gamma^{2}}+2 l_{1} \frac{1}{\gamma}
$$


It is important to note that, at this stage, the action principle (34) is completely equivalent to action (31) because the coefficients of the transformation $b_{1}$ and $b_{2}$ satisfy the condition (11).

In order to eliminate the term with $\phi_{I J}^{I J}$ in the action (34), $a_{1}$ and $a_{2}$ must satisfy the condition (22) which fixes the constants $A_{1}$ and $A_{2}$ to

$$
A_{1}=0, \quad \text { and } \quad A_{2}=\frac{a_{1} \gamma \sigma}{4},
$$

while the constants $K_{1}$ and $K_{2}$ do not get modified. Using the values for $A_{1}$ and $A_{2}$ the action principle (34) takes the form

$$
\begin{aligned}
S[B, A, \phi, \mu]= & \int_{\mathcal{M}^{4}}\left[\left(B^{I J}+\frac{1}{\gamma}{ }^{*} B^{I J}\right) \wedge F_{I J}[A]-\frac{1}{2} \phi_{I J K L} B^{I J} \wedge B^{K L}\right. \\
& \left.-\mu\left(\frac{a_{1} \gamma \sigma}{4} \phi_{I J K L} \varepsilon^{I J K L}-\lambda\right)+K_{1} B_{I J} \wedge B^{I J}+K_{2} B_{I J} \wedge^{*} B^{I J}\right],
\end{aligned}
$$

and $\lambda$ becomes

$$
\lambda=a_{1}\left[3 ! \gamma K_{2}+\frac{\Lambda}{\beta}\right] .
$$

Because of the restriction (22), the action principle (35) is a particular case of (34) and thus a particular case of (31).

In order to write the action principle (35) in terms of the tetrad and a connection we can follow two approaches. In the first, we simply use the form for the $B$ 's obtained in (27) and the value of $\lambda$ given in (36) and insert them into (35) to get

$$
\begin{aligned}
& \text { (i) } S[e, A]=\alpha \int_{\mathcal{M}^{4}}\left[\left(*\left(e^{I} \wedge e^{J}\right)+\frac{\sigma}{\gamma} e^{I} \wedge e^{J}\right) \wedge F_{I J}[A]-\frac{\Lambda}{12} \varepsilon_{I J K L} e^{I} \wedge e^{J} \wedge e^{K} \wedge e^{L}\right], \\
& \text { (ii) } S[e, A]=\alpha \int_{\mathcal{M}^{4}}\left[\left(^{*}\left(e^{I} \wedge e^{J}\right)+\gamma e^{I} \wedge e^{J}\right) \wedge F_{I J}[A]-\frac{\Lambda}{12} \varepsilon_{I J K L} e^{I} \wedge e^{J} \wedge e^{K} \wedge e^{L}\right],
\end{aligned}
$$

which is the Holst action principle with cosmological constant. Note that each solution involves a different Immirzi parameter.

In the second approach, we start from the action principle (35) where now $\lambda$ is not fixed and we simply solve the constraint on the $B$ 's that comes from (35). The relation of $\lambda$ with the cosmological constant will be obtained at the end of the procedure. The equation of motion that comes from the variation of (35) with respect to the field $\phi_{I J K L}$ implies

$$
\frac{1}{2} B_{I J} \wedge B_{K L}=-\frac{\mu a_{1} \gamma \sigma}{4} \varepsilon_{I J K L},
$$

and therefore

$$
B_{I J} \wedge B^{I J}=0, \quad B_{I J} \wedge^{*} B^{I J}=-3 ! \mu a_{1} \gamma
$$

From which we obtain the two-forms $B$ 's given by

$$
\text { (i) } B^{I J}=\kappa_{1}^{*}\left(e^{I} \wedge e^{J}\right) \quad \text { and } \quad \text { (ii) } \quad B^{I J}=\kappa_{2}\left(e^{I} \wedge e^{J}\right) .
$$

Using equations (37) and (38) we obtain

$$
\begin{aligned}
(i) \quad S[e, A]= & \kappa_{1} \int_{\mathcal{M}^{4}}\left[\left(*\left(e^{I} \wedge e^{J}\right)+\frac{\sigma}{\gamma} e^{I} \wedge e^{J}\right) \wedge F_{I J}[A]\right. \\
& \left.+\frac{\kappa_{1} \sigma}{2}\left(K_{2}-\frac{1}{3 ! \gamma} \frac{\lambda}{a_{1}}\right) \varepsilon_{I J K L} e^{I} \wedge e^{J} \wedge e^{K} \wedge e^{L}\right],
\end{aligned}
$$




$$
\begin{aligned}
\text { (ii) } S[e, A]= & \frac{\kappa_{2}}{\gamma} \int_{\mathcal{M}^{4}}\left[\left({ }^{*}\left(e^{I} \wedge e^{J}\right)+\gamma e^{I} \wedge e^{J}\right) \wedge F_{I J}[A]\right. \\
& \left.+\frac{\kappa_{2} \gamma}{2}\left(K_{2}-\frac{1}{3 ! \gamma} \frac{\lambda}{a_{1}}\right) \varepsilon_{I J K L} e^{I} \wedge e^{J} \wedge e^{K} \wedge e^{L}\right] .
\end{aligned}
$$

These actions have the form of the Holst's action principle with cosmological constant $\Lambda$ given in each case by

$$
\text { (i) } \frac{\Lambda}{12}=-\frac{\kappa_{1} \sigma}{2}\left(K_{2}-\frac{1}{3 ! \gamma} \frac{\lambda}{a_{1}}\right), \quad \text { (ii) } \frac{\Lambda}{12}=-\frac{\kappa_{2} \gamma}{2}\left(K_{2}-\frac{1}{3 ! \gamma} \frac{\lambda}{a_{1}}\right) .
$$

They fix the relationship among the constants $\lambda, \gamma$, and $a_{1}$ that appear in the action principle (35), and the coefficients of the solutions given in (38). It is easy to see that, for each case, $\lambda$ has the form

$$
\text { (i) } \lambda=a_{1}\left[3 ! \gamma K_{2}+\frac{\Lambda \sigma \gamma}{\kappa_{1}}\right], \quad(i i) \quad \lambda=a_{1}\left[3 ! \gamma K_{2}+\frac{\Lambda}{\kappa_{2}}\right],
$$

respectively. Note that in order for these values of $\lambda$ match the value of $\lambda$ given in (36) (i.e. the two approaches give the same result), it is required that $\kappa_{1}=\sigma \gamma \beta$ and $\kappa_{2}=\alpha \gamma$, respectively. These are the same values obtained for $\kappa_{1}$ and $\kappa_{2}$ in the analysis after equation (27).

\section{Conclusions}

It has been shown that by performing an invertible transformation of the fields of the BF formulation for general relativity given by Capovilla, Montesinos, Prieto, and Rojas [12], it is possible to obtain the action principle (19) which includes the two BF terms $B^{I J} \wedge F_{I J}$ and ${ }^{*} B^{I J} \wedge F_{I J}$ and still involves the two Lorentz invariants $\phi_{I J K L} \varepsilon^{I J K L}$ and $\phi_{I J}{ }^{I J}$ generically. One of the results of the analysis is to clearly show the relationship of the two parameters $a_{1}$ and $a_{2}$ of the CMPR action principle and of the two parameters $b_{1}$ and $b_{2}$ involved in the transformation with the Immirzi parameter.

From the analysis is clear that the freedom in the choice of the parameters $a_{1}, a_{2}, b_{1}$, and $b_{2}$ can be used to handle the two Lorentz invariants $\phi_{I J K L} \varepsilon^{I J K L}$ and $\phi_{I J}{ }^{I J}$ that appear in the transformed action principle. In particular, a suitable combination of these parameters can result in that one of these invariants is missing in the transformed action principle as it is shown in the Section 3.1. As an application of this fact, the action used in [17] is obtained in Section 3.1.1.

Finally, and as another application of the transformation discussed in this paper, the coupling of the cosmological constant to the action principle used in [17] is obtained from the coupling of the cosmological constant to the CMPR action principle studied in [18].

\section{Acknowledgements}

This work was partially supported by Conacyt, grant number 56159-F.

\section{References}

[1] Ashtekar A., Lectures on non-perturbative canonical gravity (Notes prepared in collaboration with Ranjeet S. Tate), Advanced Series in Astrophysics and Cosmology, Vol. 6, World Scientific Publishing Co., Inc., River Edge, NJ, 1991.

Thiemann T., Modern canonical quantum general relativity, Cambridge Monographs on Mathematical Physics, Cambridge University Press, Cambridge, 2007.

Rovelli C., Quantum gravity, Cambridge Monographs on Mathematical Physics, Cambridge University Press, Cambridge, 2004. 
Ashtekar A., Lewandowski J., Background independent quantum gravity: a status report, Classical Quantum Gravity 21 (2004), R53-R152, gr-qc/0404018.

Perez A., Introduction to loop quantum gravity and spin foams, in Proceedings of 2nd International Conference on Fundamental Interactions (Domingos Martins, Brazil, June 6-12, 2004), Editora Rima, Sao Paulo, 2004, 221-295, gr-qc/0409061.

Rovelli C., Loop quantum gravity, Living Rev. Relativ. 11 (2008), no. 5, 69 pages.

[2] Perez A., Spin foam models for quantum gravity, Classical Quantum Gravity 20 (2003), R43-R104, gr-qc/0301113.

Oriti D., Spacetime geometry from algebra: spin foam models for non-perturbative quantum gravity, Rep. Progr. Phys. 64 (2001), 1703-1757, gr-qc/0106091.

Baez J.C., An introduction to spin foam models of BF theory and quantum gravity, in Geometry and Quantum Physics (Schladming, 1999), Lecture Notes in Phys., Vol. 543, Springer, Berlin, 2000, 25-93, gr-qc/9905087.

Baez J.C., Spin foam models, Classical Quantum Gravity 15 (1998), 1827-1858, gr-qc/9709052.

Engle J., Pereira R., Rovelli C., Loop-quantum-gravity vertex amplitude, Phys. Rev. Lett. 99 (2007), 161301, 4 pages, arXiv:0705.2388.

Livine E.R., A short and subjective introduction to the spinfoam framework for quantum gravity, arXiv:1101.5061.

[3] Alexandrov S., The new vertices and canonical quantization, arXiv:1004.2260.

Rovelli C., Speziale S., Lorentz covariance of loop quantum gravity, Phys. Rev. D 83 (2011), 104029, 6 pages, arXiv:1012.1739.

[4] Plebański J.F., On the separation of Einsteinian substructures, J. Math. Phys. 18 (1977), 2511-2520.

[5] Samuel J., A Lagrangian basis for Ashtekar's reformulation of canonical gravity, Pramana J. Phys. 28 (1987), L429-L432.

[6] Jacobson T., Smolin L., The left-handed spin connection as a variable for canonical gravity, Phys. Lett. B 196 (1987), 39-42.

Jacobson T., Smolin L., Covariant action for Ashtekar's form of canonical gravity, Classical Quantum Gravity 5 (1988), 583-594.

[7] Reisenberger M.P., New constraints for canonical general relativity, Nuclear Phys. B 457 (1995), 643-687, gr-qc/9505044.

[8] Capovilla R., Dell J., Jacobson T., A pure spin-connection formulation of gravity, Classical Quantum Gravity 8 (1991), 59-73.

[9] Robinson D.C., A Lagrangian formalism for the Einstein-Yang-Mills equations, J. Math. Phys. 36 (1995), 3733-3742.

[10] Reisenberger M.P., Classical Euclidean general relativity from 'left-handed area = right-handed area', Classical Quantum Gravity 16 (1999), 1357-1371, gr-qc/9804061.

[11] De Pietri R., Freidel L., so(4) Plebański action and relativistic spin-foam model, Classical Quantum Gravity 16 (1999), 2187-2196, gr-qc/9804071.

[12] Capovilla R., Montesinos M., Prieto V.A., Rojas E., BF gravity and the Immirzi parameter, Classical Quantum Gravity 18 (2001), L49-L52, gr-qc/0102073.

[13] Barbero J.F., Real Ashtekar variables for Lorentzian signature space-times, Phys. Rev. D 51 (1995), 55075510, gr-qc/9410014.

[14] Immirzi G., Real and complex connections for canonical gravity, Classical Quantum Gravity 14 (1997), L177-L181, gr-qc/9612030.

[15] Holst S., Barbero's Hamiltonian derived from a generalized Hilbert-Palatini action, Phys. Rev. D 53 (1996), 5966-5969, gr-qc/9511026.

[16] Livine R.E., Oriti D., Barrett-Crane spin foam model from generalized BF-type action for gravity, Phys. Rev. D 65 (2002), 044025, 12 pages, gr-qc/0104043.

[17] Engle J., Pereira R., Rovelli C., Flipped spinfoam vertex and loop gravity, Nuclear Phys. B 798 (2008), 251-290, arXiv:0708.1236.

[18] Montesinos M., Velázquez M., BF gravity with Immirzi parameter and cosmological constant, Phys. Rev. D 81 (2010), 044033, 4 pages, arXiv:1002.3836.

[19] Velázquez M., BF gravity, matter couplings, and related theories, Ph.D. Thesis, Cinvestav, Mexico, 2011.

[20] Cuesta V., Montesinos M., Cartan's equations define a topological field theory of the BF type, Phys. Rev. D 76 (2007), 104004, 6 pages. 
[21] Liu L., Montesinos M., Perez A., Topological limit of gravity admitting an SU(2) connection formulation, Phys. Rev. D 81 (2010), 064033, 9 pages, arXiv:0906.4524.

[22] Magaña R., Análisis hamiltoniano del término agregado por Holst a la lagrangiana de Palatini, M.Sc. Thesis, Cinvestav, Mexico, 2007.

[23] Liu L., Analyse Hamiltonienne des Théories des champs invariantes par difféomorphismes, Memoire de Master 2, Centre de Physique Theorique de Luminy, Marseille, France, 2009.

[24] Montesinos M., Genuine covariant description of Hamiltonian dynamics, in Proceedings of VI Mexican School on Gravitation and Mathematical Physics "Approaches to Quantum Gravity" (Playa del Carmen, Mexico, November 21-27, 2004), Editors M. Alcubierre, J.L. Cervantes-Cota, M. Montesinos, J. Phys.: Conf. Ser. 24 (2005), 44-51, gr-qc/0602072.

[25] Montesinos M., Alternative symplectic structures for $\mathrm{SO}(3,1)$ and $\mathrm{SO}(4)$ four-dimensional $\mathrm{BF}$ theories, Classical Quantum Gravity 23 (2006), 2267-2278, gr-qc/0603076.

[26] Smolin L., Speziale S., A note on the Plebanski action with cosmological constant and an Immirzi parameter, Phys. Rev. D 81 (2010), 024032, 6 pages, arXiv:0908.3388. 